\title{
Multi-ion sensing device for horticultural application based upon chemical modification and special packaging of ISFETs
}

\author{
H. H. van den Vlekkert, U. H. Verkerk, P. D. van der Wal and A. van Wingerden \\ Priva B.V., P.O. Box 18, 2678 ZG De Lier (Netherlands)
}

D. N. Reinhoudt and J. R. Haak

Faculty of Chemical Technology, University of Twente, 7500 AE Enschede (Netherlands)

G. W. N. Honig and H. A. J. Holterman

Twente Transfer Technology B.V., P.O. Box 3639.7500 DP Enschede (Netherlands)

\begin{abstract}
A multi-ion sensing device, based on ISFETs, is described for horticultural applications. The advantages of a new packaging technique based on back-side contacted ISFETs are outlined. The basic pH-sensitive ISFETs can be modified to, e.g., potassium-sensitive devices by using neutral carrier-based membranes and an intermediate hydrogel. Both membrane and hydrogel are chemically anchored to the ISFET surface and the ionophore is chemically bonded to the membrane. It is shown that these chemical modifications of the membrane system lead to an increased lifetime for the sensor.
\end{abstract}

\section{Introduction}

The first developments concerning the growth on a commercial scale of horticultural crops using an artificial medium rather than soil took place in Scandinavia [1]. Since 1980, in particular in the Netherlands, the area of crops on substrate has increased rapidly, especially for the growth of vegetables. The reasons for this remarkable shift are the urge to avoid infection of the soil with highly toxic and expensive chemicals and to increase the production and quality of the crop.

The rockwool system has one major disadvantage. To achieve optimal growth conditions an excess of water has to be given to the plants. There are three reasons for this surplus: the substantial difference in transpiration between plants, the uneven supply of water per plant due to the inaccuracy of the trickle irrigation system and, last but not least, accumulation of sodium and chloride or even certain nutrients in the rockwool slabs, which has to be avoided [1]. This excess of water containing nutrients (drainage water) flows freely to the surface water. Apart from the loss of raw materials, this causes considerable pollution of the environment. This form of environmental pollution and loss of fertilizers and water can be pre- vented by collecting the drainage water in a gutter system and reusing it.

However, the composition of this drainage water is unknown and therefore precise control of the nutrients in the irrigation water (a mixture of fresh water and drainage water) is impossible. Since the plant-uptake of nutrients can vary considerably [2], continuous measurement of the drainage water composition is preferable to discontinuous analysis from samples. This monitoring of the drainage water composition can also be used to follow the accumulation of sodium and chloride and initiate a warning signal to prevent damage to the crop. An ideal closed-loop system is shown in Fig. 1. Furthermore, information on plant-uptake, determined from irrigation water and drainage water composition, could lead to a better understanding of plant physiology and thereby improved control of plant growth, higher production and better quality. Although research has been done with conventional ion-selective electrodes [3], no continuous measurement system has been developed that is compatible with the demands of the application of sensors in horticulture.

In principle an ISFET-based system could fulfil the harsh horticulture requirements (lifetime $>1$ 


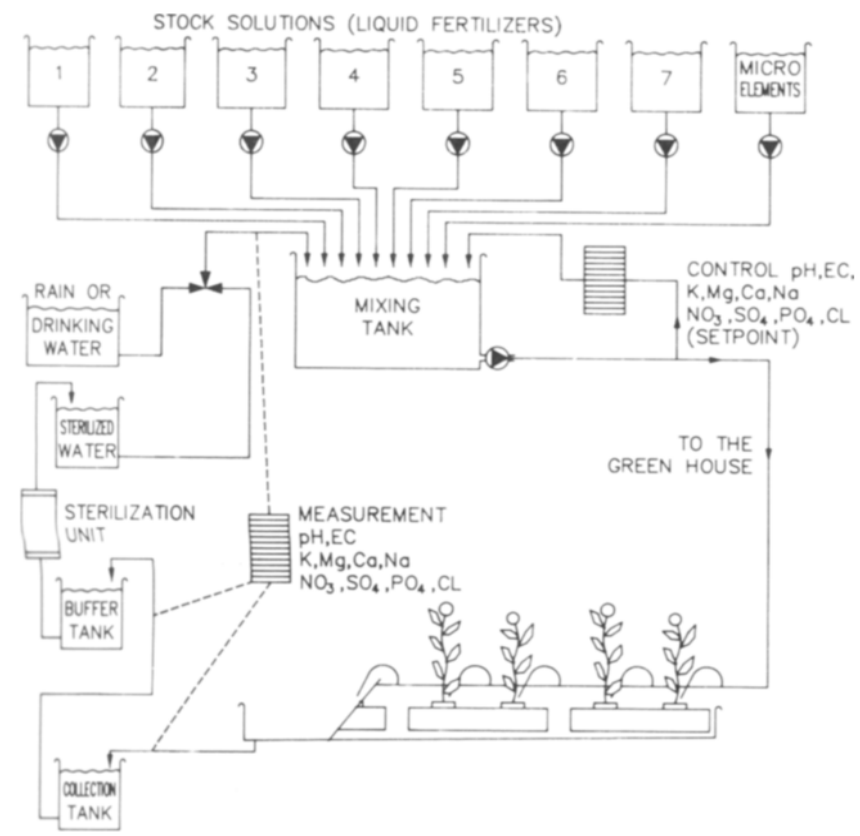

Fig. 1. Schematic representation of a closed-loop system.

year, accuracy $<10 \%$ and low maintenance level). In order to ensure a long lifetime, both ISFET packaging and the organic membranes used to change the sensitivity have to be improved. These requirements mean that an automatic calibration system with low consumption of solutions has to be part of the measurement set-up.

\section{Experimental}

The packaging problem is solved by using plastic encased back-side contacted ISFETs [4], with alumina as the pH-sensitive layer. The electrical contacts are isolated from the solution by means of O-rings, thereby circumventing the use of resins to establish electrical isolation from the analyte solution (Fig. 2). A multi-ion flow-through cell is constructed by placing individual sensors adjacent to each other, also by using O-ring connections.

The application of polymeric membranes to modify the sensitivity of planar sensors introduces some problems, like the fixation of these membranes on the planar ISFETs, the necessity of an intermediate hydrogel [5] and the limited lifetime due to leaching out of the membrane components [6].

The membrane problems with respect to fixation are solved by covalently connecting the hydrogel

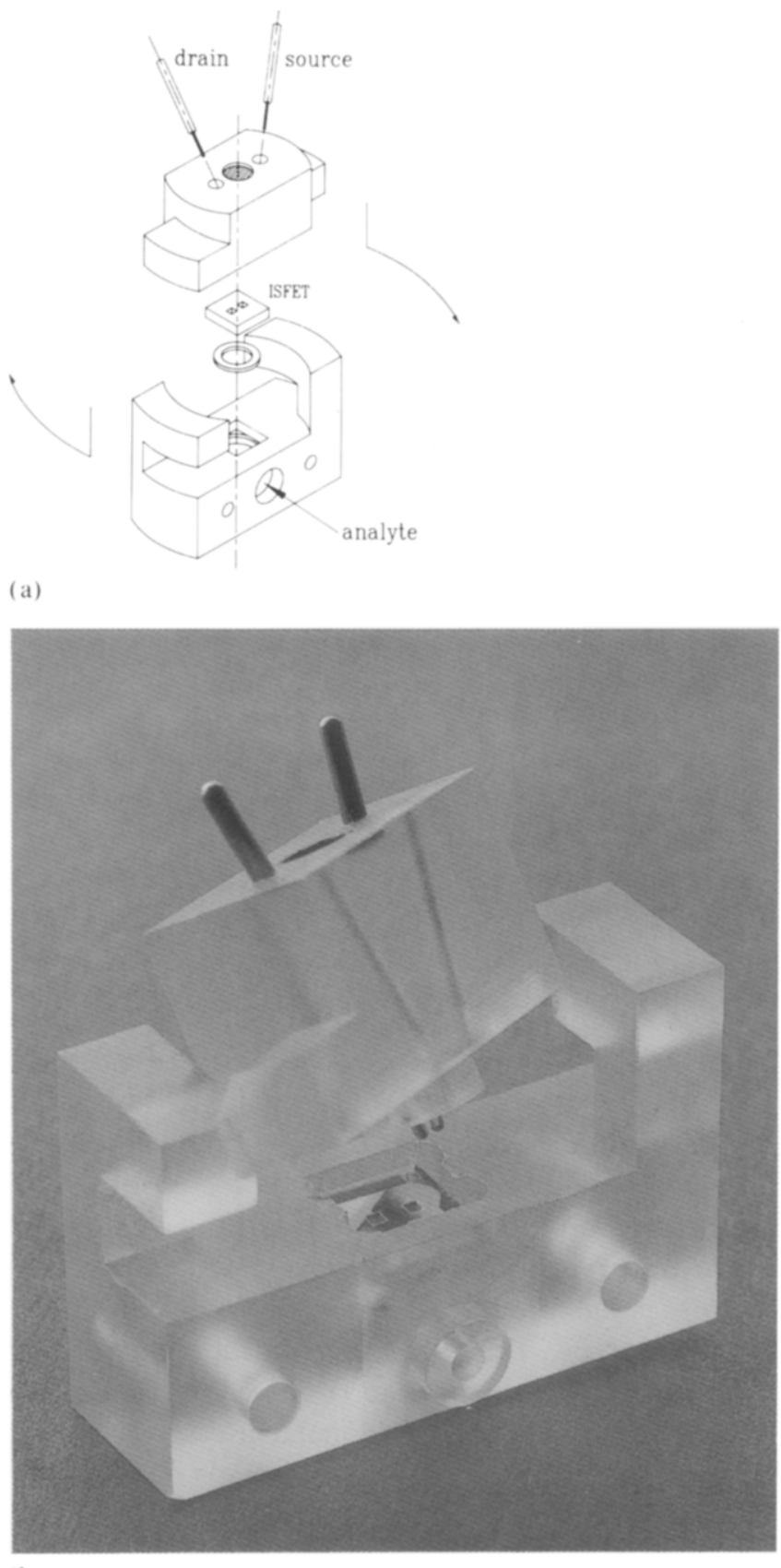

(b)

Fig. 2. (a) Schematic drawing of the packaging. (b) Single packaging unit.

and ion-sensitive membrane both to each other and to the sensor surface $[7,8]$. The leaching out of the ionophore from the membrane face is prevented by chemical anchoring to the membrane. Potassium and calcium sensors based on the above concepts have been developed [9].

The ISFETs are hooked up to a system which operates the ISFETs in the constant-current mode whereby either analyte or one out of two calibra- 


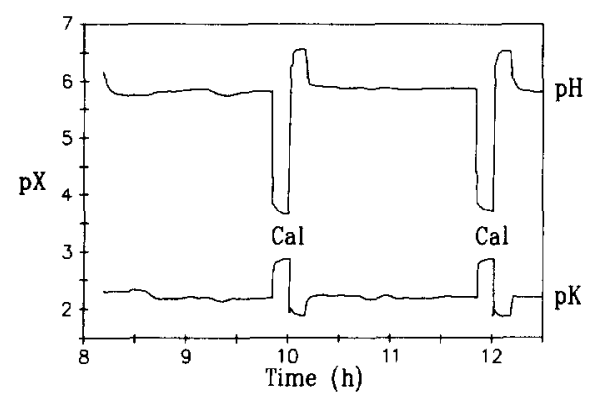

Fig. 3. Typical $\mathrm{pH}$ and $\mathrm{pK}$ measurements in nutrient solution with regular two-point calibration (cal).

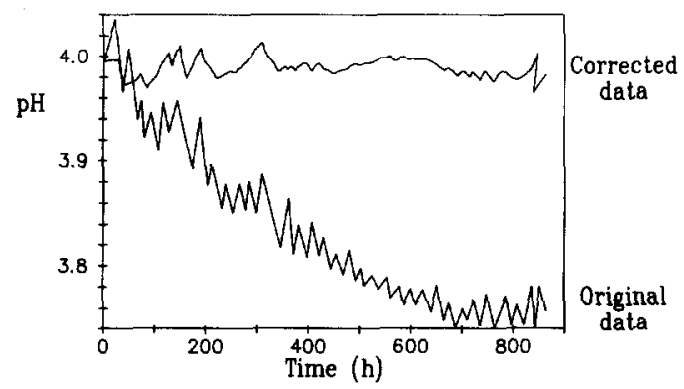

Fig. 4. The effect of output correction for drift and temperature changes on $\mathrm{pH}$ sensors with respect to one calibration solution.

tion solutions can be measured automatically. In the greenhouse (tomato culture), irrigation water is continuously measured and every two hours a two-point calibration is carried out. From these calibration points the sensitivity, drift and temperature sensitivity can be calculated (a temperature sensor is included in the flow-through cell). The outputs of the ISFETs (in $\mathrm{mV}$ ) are transformed into $\mathrm{pH}$ and potassium activities by means of an algorithm [10], using the calculated sensitivity, drift and temperature sensitivity. With this algorithm the effects of temperature and drift can be corrected (Figs. 3 and 4). The experiments are performed with pH-ISFETs and potassium ISFETs with both covalently attached (anchored) and non-covalently attached (free) ionophore.

\section{Results and discussion}

The pH-ISFETs have been tested for more than 6 months in the greenhouse. Before this test they had already been used for laboratory experiments intermittently for over four months. Over the whole period no change in sensitivity was detected $(53 \mathrm{mV} / \mathrm{pH})$. Using the algorithm to calculate the

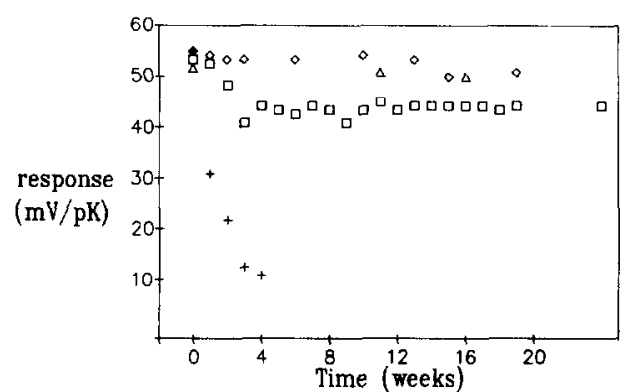

Fig. 5. Sensitivity of potassium sensors under continuous flow conditions for: + , free ionophore (in the greenhouse); $\square$, anchored ionophore (in the greenhouse); $\diamond$, anchored ionophore (laboratory); $\triangle$, anchored ionophore (in the greenhouse with $\mathrm{KCl} / \mathrm{NaCl}$ solutions).

$\mathrm{pH}$, an overall accuracy of better than $0.02 \mathrm{pH}$ units over a period $>1$ month can be achieved without recalibration. These results indicate that the drift and temperature sensitivity of pH-ISFETs are very stable over long periods of time. The recalibration frequency can therefore be low and consumption of the calibration solutions can be kept to a minimum.

The time dependence of the sensitivity of the potassium sensors is shown in Fig. 5. It can be seen that the sensor with free ionophore loses its sensitivity very rapidly. For the sensor with anchored ionophore, a small decrease is observed in the first weeks, after which the sensitivity remains stable over several months. In contrast, under laboratory conditions no decay of sensitivity was detected for the anchored ionophore. It should be noted that, in the greenhouse, the sensitivity was determined in mixed solutions containing different salts. For the laboratory experiments only solutions containing $\mathrm{KCl}$ and $\mathrm{NaCl}$ are used. To investigate this discrepancy the sensitivity of the potassium sensors in the greenhouse was determined from time to time with the same solutions as used for the laboratory experiments. Using these solutions, no decay in sensitivity is detected. The reason for this difference has to be related to changes in the ion-selective membrane, such as leaching out of anionic sites. Experiments to clarify this phenomenon are in progress.

\section{Conclusions}

It has been shown that both ISFET packaging and ion-selective membranes can be improved to 
meet the needs for horticultural applications. Thus when ISFETs for other ions become available, a continuous control of irrigation and drainage water can be realized in the near future. Even a system containing only $\mathrm{pH}$, potassium, calcium and sodium sensors can already be an important improvement with respect to the existing system based on $\mathrm{pH}$ and electrical conductivity for irrigation water control.

Apart from the specific horticultural application, the basic packaging design is also suitable for flow-injection analysis systems (minimum sample volume of a single sensor is $2.5 \mu \mathrm{l}$ ). Due to this construction, the choice of ion-sensitive, enzyme-sensitive or gas-permeable membranes, which can be used to change the sensitivity of the basic pH-ISFET, becomes nearly unlimited. The membranes do not have to be compatible with any kind of epoxy and, for on-wafer production, the final membrane layer is not subject to any photolithographic procedure which would be necessary if the contacts were located at the same side as the gate area. A practical advantage is that upon malfunction, the sensor can be replaced in a few seconds and the housing can be reused.

\section{Acknowledgements}

The authors acknowledge the technical support received from Sentron (Roden, The Netherlands) and the University of Neuchâtel (Neuchâtel,
Switzerland). Financial support was received from CME Twente and StiPt.

\section{References}

1 C. Sonneveld, Rockwool as a substrate in protected cultivation, Proc. Horticulture in High Technology Era, Tokyo, Japan, 1988, pp. $173-179$.

$2 \mathrm{~W}$. Voogt, Recirculatie systemen: kringloopprincipe in de substraatteelt, Landbouwk. Tijdschr., 100(8) (1988) 29-31.

3 W. J. Albery, H. J. J. Drummond, N. J. Freeman, B. G. D. Haggett, C. C. Jones, C.P. Jones and M. D. Neville, Chemical sensors and instrumentation for horticulture, Proc. Horticulture in High Technology Era, Tokyo, Japan, 1988, pp. 195-201.

4 H. H. van den Vlekkert, B. Kloeck, D. Prongue, J. Berthoud, B. $\mathrm{Hu}, \mathrm{N}$. F. de Rooij, E. Gilli and Ph. de Crousaz, A pH-ISFET and an integrated $\mathrm{pH}$-pressure sensor with back-side contacts, Sensors and Actuators, 14 (1988) 165-176.

5 H. H. van den Vlekkert, A van den Berg, A. Grisel and N. F. de Rooij, Solvent polymeric membranes combined with chemical solid-state sensors, Analyst, 113 (1988) 1029-1033.

6 O. Dinten, U. E. Spichter, N. Chaniotakis, P. Gehrig, B. Rusterholz, W. E. Morf and W. Simon, Lifetime of neutral-carrier base liquid membranes in aqueous samples and blood and the lipophilicity of membrane components, Anal. Chem., 63 (1991) 596-603.

7 E. J. R. Sudholter, P. D. van der Wal, M. Skowronska-Ptasinska, A. van den Berg, P. Bergveld and D. N. Reinhoudt, Modification of ISFETs by covalent anchoring of poly(hydroxyethyl methacrylate) hydrogel, Anal. Chim. Acta, 230 (1990) 59-65.

8 D. N. Reinhoudt and E. J. R. Sudholter, The transduction of host-guest interactions into electronic signals by molecular systems, Adv. Mater., 2 (1990) 23-32.

9 H. A. J. Holterman, G. W. N. Honig, E. J. R. Sudholter, D. N. Reinhoudt and H. H. van den Vlekkert, Chemically modified ISFETs for the selective sensing of potassium and calcium in mixed clectrolyte solution, Proc. 3rd Int. Meet. Chemical Sensors, Cleveland, $O H, U S A, 1990$, p. 40.

$10 \mathrm{H}$. H. van den Vlekkert and N. F. de Rooij, Design, fabrication and characterization of pH sensitive ISFETs, Analusis, 16(2) (1988) $110-119$. 